\title{
Evolution et perspectives de la demande intérieure de corps gras en Afrique subsaharienne
}

\section{Patterns and prospects in domestic demand for fats and oils in sub-Saharan Africa}

Oléagineux, Corps Gras, Lipides. Volume 7, Numéro 2, 147-54, Mars - Avril 2000, Dossier : Afrique, plantation et développement

\section{Auteur(s) : Robert HIRSCH}

Résumé : Si les corps gras peuvent constituer l'une des clés de voûte de l'agriculture de certains pays émergents (Malaisie, Brésil), ils ne font l'objet, en Afrique, que de très peu d'intérêt. Rarement cités parmi les produits de première nécessité, éclatés entre différentes filières (palme, coton, arachide), les corps gras ne se situent au cœur des préoccupations des décideurs qu'en période de crise tandis que, de leur côté, les bailleurs de fonds ne leur portent qu'une attention modérée, le plus souvent ponctuelle et sans vision stratégique. Dans les années 60 , c'est-à-dire à l'époque des indépendances, le continent africain disposait pourtant de sérieux atouts, puisqu'il dominait largement le marché mondial des produits du palmier (huile et palmistes) et était encore bien placé sur celui des produits de l'arachide (huile et tourteaux). Des pays comme le Nigeria ou l'ex-Zaïre disposaient d'un potentiel alors jugé prometteur, apte à conforter leurs positions commerciales. Mais le pétrole pour le premier et les crises politiques à répétition pour le second ont assez rapidement réduit à néant les espoirs que l'on pouvait légitimement placer dans l'agriculture de ces pays. $\mathrm{Si}$, à partir des années 70 , le soja, en Amérique latine, et l'huile de palme, en Asie du Sud-est, ont réussi à s'imposer sur un marché mondial en pleine expansion, l'Afrique, qui n'était dépourvue ni de terres, ni de main-d'œuvre, ni des acquis d'une recherche globalement performante, s'est repliée sur elle-même. Les investissements non négligeables, réalisés dans un premier temps en Côte d'Ivoire, au Cameroun et dans quelques autres pays, étaient financés par les États eux-mêmes à travers des sociétés publiques souscapitalisées et rarement gérées dans une perspective de maîtrise des coûts, à qui était assigné un objectif prioritaire de satisfaction des besoins intérieurs, dénué de réelles ambitions continentales ou mondiales. Dans les années 90, les États ont été relayés par un mouvement largement spontané de plantations villageoises ou de taille moyenne, mais sans qu'un véritable suivi des superficies et du matériel végétal utilisé ne soit mis en place et permette d'en apprécier l'ampleur et l'impact. De surcroît, la déconnexion quasi totale de ces filières oléagineuses africaines - anciennes ou nouvelles - vis-à-vis du marché mondial et les remparts longtemps érigés par leurs tutelles - prix administrés, forte protection aux frontières, faible autonomie des responsables des unités de production - ont, simultanément, affecté leur dynamisme, pénalisé les consommateurs et empêché l'organisation et l'émergence d'acteurs privés capables de s'implanter sur un marché mondial des corps gras d'origine végétale en expansion continue depuis le milieu du xxe siècle.

Summary : Smallholders schemes started in the Seventies, with the help of Public Funds. Twenty years later, a new dynamics can be observed in Cameroon. Due to several factors - the economic crisis, falling salaries, a decline in cocoa purchase prices, etc. - this movement has been impulse by new actors, mainly urban, mostly non estate residents and poorly organized. Many of them produce and sell crude palm oil instead of ffb. Beyond this apparent success - all investment requirements are self-financed, without any bank loans - strong competition between smallholders, large oil mills and 
imported oils can be predicted in a near future. There is an urgent need for a new organization of all the partners involved in the palm oil sector.

Keywords : palm oil, smallholdings, development, organization.

\section{ARTICLE}

\section{Déterminants de la demande intérieure de corps gras en Afrique}

Au-delà des indications précieuses que donne, par exemple, la consommation par tête de corps gras des pays africains, il est particulièrement difficile de caractériser cette demande qui est à la fois :

- multiforme, puisqu'elle concerne l'alimentation humaine et animale, mais aussi l'industrie, la savonnerie, la cosmétologie ;

- volatile, car sujette à une très forte substituabilité entre les huiles raffinées dont le prix est souvent un facteur déterminant ;

- complexe, du fait de l'existence de coproduits ou de sous-produits qui peuvent influencer la formation du prix.

Ces difficultés, bien connues, ne sont pas les seules à rendre délicate l'analyse de la demande. Une infinité de facteurs peuvent aussi expliquer et donc caractériser la demande de corps gras dans un pays donné à une période donnée et, parmi eux, sans prétendre être exhaustif, on peut retenir les facteurs culturels et sociaux, démographiques, économiques et monétaires et, enfin, politiques et institutionnels.

\section{Facteurs culturels et sociaux}

De nombreux pays et de vastes zones, en Afrique, ne consomment que très peu de corps gras non seulement parce que l'offre est inexistante, mais aussi parce que les habitudes alimentaires n'intègrent pas ces corps gras dans la pratique quotidienne. Madagascar et certains pays d'Afrique de I'Est sont représentatifs d'une telle situation qui interdit toute recherche d'une corrélation entre les divers indices de pauvreté ou de développement, calculés par les Nations unies, et la consommation de corps gras.

\section{Facteur démographique}

Il pèse à la fois sur la demande actuelle de nombreux pays peu peuplés et/ou à faible densité et sur l'avenir puisque l'inflexion des taux de croissance de nombreux pays est désormais perceptible et devrait les faire entrer dans ce que les spécialistes appellent la " transition démographique ». En d'autres termes, les prévisions des organismes internationaux, souvent alarmistes, doivent être relativisées et faire l'objet de corrections.

\section{Facteurs économiques et monétaires}

Ils influencent, directement ou indirectement, la demande globale, qu'il s'agisse du produit intérieur brut ou de la recherche de l'équilibre de la balance commerciale ${ }^{1}$. Sur le plan micro-économique, 
l'évolution des prix, des revenus et donc du pouvoir d'achat influence également la demande de corps gras, même si les habitudes alimentaires locales peuvent perturber l'établissement d'un lien de cause à effet durable entre hausse des revenus et consommation de corps gras. Néanmoins, la croissance spectaculaire de la demande de corps gras de pays émergents aussi différents que la Chine, l'Indonésie, le Brésil ou le Mexique ${ }^{2}$ semble indiquer que la liaison entre revenu et demande de corps gras est d'autant plus forte que le seuil de saturation n'est pas atteint ${ }^{3}$.

Le taux de change d'un pays et son endettement peuvent également affecter la demande, particulièrement lorsque la satisfaction de cette dernière nécessite des importations significatives de corps gras.

\section{Facteurs politiques et institutionnels}

Très variés eux aussi, ils peuvent englober l'existence ou l'absence d'une stratégie nationale de développement des corps gras, du contenu et de l'envergure de celle-ci (nationale, régionale ou mondiale), du degré de protection du marché intérieur, de l'ampleur du désengagement de l'État des activités de production, de la capacité des producteurs à s'organiser et à défendre leurs intérêts.

En résumé, l'estimation de la demande actuelle et future est confrontée à un grand nombre d'incertitudes que l'absence - ou du moins la rareté - d'enquêtes périodiques de consommation rend difficiles à éliminer.

\section{Demande africaine de corps gras}

Pas plus pour les corps gras que pour tout autre groupe de produits d'origine agricole, l'Afrique n'est homogène. Quantifier la demande suppose donc que l'on s'entende d'abord sur la définition de I'Afrique que l'on souhaite étudier : Afrique OUA (53 pays), Afrique subsaharienne (48 pays) et divers regroupements régionaux (CEDEAO, COMESA, CEEAC, SADC, IGAD, COI) coexistent et se superposent, les uns fondés sur des réalités géopolitiques, les autres ayant une connotation essentiellement politique sans rapport avec notre propos.

Dans un souci de simplicité et bien que l'Afrique au nord du Sahara ne présente que très peu de similitudes avec le reste du continent, on retiendra dans un premier temps I'Afrique OUA pour analyser la situation des corps gras dans leur globalité. Ensuite, les différentes zones et les différents corps gras seront examinés de manière plus détaillée, étant entendu que la demande globale satisfaite ou non - ne peut être dissociée de l'offre et des échanges.

Demande de corps gras à l'échelle de l'OUA

L'ambiguïté du terme est réelle : s'agit-il de la demande potentielle de corps gras et, si oui, selon quelle norme (nord-américaine, avec près de $50 \mathrm{~kg}$ par tête et par an ou asiatique avec $15 \mathrm{~kg}$ ) ou s'agit-il de consommations effectives, tous usages confondus, que l'on peut estimer par le biais des disponibilités apparentes (production + importations - exportations \pm variation des stocks) ? La réponse n'est pas simple dans la mesure où c'est l'appareil statistique existant qui, seul, permet de choisir. Et cet appareil statistique, pour l'Afrique, est particulièrement rudimentaire, comme en témoignent les difficultés d'élaboration, pour un pays donné, d'un simple tableau ressources/emplois retraçant les flux physiques des divers corps gras. Les sources nationales, par FAO interposée, indiqueront par exemple la production de graines oléagineuses par pays, mais sans en préciser les 
utilisations finales (ventes à une huilerie, fabrication domestique d'huile artisanale, exportations en l'état, semences, pertes, etc.), tandis qu'Oil World ne s'intéresse qu'aux pays réellement insérés dans les échanges mondiaux (Sénégal, Nigeria).

Pour les échanges, qui permettent de compenser aussi bien les insuffisances que les excès de l'offre, les mêmes difficultés existent et sont encore amplifiées par l'extrême diversité des produits, de leur degré de transformation et de leur utilisation finale, diversité souvent mal prise en compte par la nomenclature douanière. Et ce qui vient d'être dit pour les oléagineux d'origine végétale s'applique également aux corps gras d'origine animale, statistiquement tout aussi difficiles à apprécier en Afrique. Par chance, ils ne jouent qu'un rôle mineur dans la demande alimentaire africaine (4 à $5 \%$ environ), qui a anticipé en quelque sorte le net déclin des corps gras animaux dans le monde (55\% de la consommation mondiale au début du $x^{e}$ siècle contre moins de $20 \%$ aujourd'hui).

Toutes les données utilisées, par pays ou agrégées, devront donc être considérées comme des ordres de grandeur.

Pour l'ensemble des corps gras végétaux et animaux, le bilan de l'Afrique OUA, établi sur la base de moyennes quinquennales, retrace, avec l'aide de quelques ratios caractéristiques, l'évolution des disponibilités, tous usages confondus, depuis les années 60 (tableau 1).

Sur une période d'environ 35 ans, que constate-t-on ?

- tout d'abord que les disponibilités apparentes ont augmenté (+ $4 \%$ par an) à un rythme supérieur à la croissance démographique $(+2,9 \%)$, ce qui semble indiquer un certain dynamisme de la demande

- que le disponible par tête et par an n'a pu augmenter (de 7,4 à 10,8 kg/an) que grâce à la croissance soutenue des importations (+ 6,8 \% par an) : celles-ci, rapportées aux disponibilités, passent en effet de 17 à $41 \%$;

- le taux d'autosuffisance en corps gras de l'Afrique s'est fortement dégradé (66 contre $113 \%$ ) et le solde des échanges, positif de plus de 300000 tonnes au début des années 60, est devenu lourdement négatif (de près de 2,8 millions de tonnes en 1996/1998) ;

- les exportations totales, stagnantes en début de période, en baisse sensible dans les années 80 , puis en léger redressement ensuite, ne représentent plus, en volume, que $10 \%$ de la production africaine de corps gras, contre $27 \%$ en 1961/1965. Encore faut-il rappeler que l'Afrique au nord du Sahara, grâce à l'huile d'olive tunisienne, assure près du quart des exportations du continent, dont elle contribue, il convient de le signaler aussi, à creuser le déficit de près de 2 millions de tonnes (soit plus de $70 \%$ du déficit de I'OUA).

\section{Demande de corps gras de l'Afrique subsaharienne (Ass)}

Pour l'Afrique subsaharienne, le même exercice conduit à des constats assez proches (tableau 2), même si le taux d'autosuffisance est, globalement, meilleur que pour l'Afrique OUA (83 contre 66 \% en 1996/1998) et même si $25 \%$ des corps gras disponibles sont importés (contre $40 \%$ ). En revanche, les importations augmentent plus rapidement et les exportations baissent également plus vite en 
ASS, ce qui traduit à la fois une croissance moindre des disponibilités globales et un disponible par tête sensiblement inférieure (8,2 contre 10,8 kg/tête en 1996/1998).

Mais situer uniquement l'Afrique subsaharienne par rapport à l'Afrique OUA n'est pas suffisant et I'hétérogénéité des habitudes alimentaires au même titre que les inégalités des niveaux de développement nous invitent à examiner comment ces divers paramètres se situent par grande zone. Pour ce faire, six régions, plus ou moins homogènes et inspirées davantage par la géographie que par tout autre critère ${ }^{4}$, ont été identifiées. Les bilans alimentaires établis périodiquement par la FAO, sur une base triennale, ont été utilisés. Le recours à cette source évite de recalculer pour 48 pays, à partir de la production et des échanges portant sur 17 corps gras majeurs (tels que définis par Oil World), les disponibilités totales, par tête et par type d'huile.

Les résultats obtenus, dans un premier temps, par pays, puis regroupés par grande zone, sont résumés dans le tableau 3 (hors utilisations non alimentaires).

Le tableau 3 confirme la forte hétérogénéité de la demande selon les zones (un rapport de 1 à 3 peut être observé), mais aussi certaines similitudes comme la prédominance des huiles végétales qui représentent entre 83,5 et $97,7 \%$ des disponibilités totales en corps gras alimentaires.

L'Afrique de l'Est et les îles de l'océan Indien se signalent par la faiblesse de la demande (satisfaite) en corps gras alimentaires. Avec 4 à $5 \mathrm{~kg}$ par tête et par an, on est en apparence très en deçà d'une couverture minimale des besoins en lipides même si, au sein de ces régions, la situation varie d'un pays à l'autre ${ }^{5}$.

L'Afrique australe et l'Afrique sahélienne occupent une position intermédiaire avec respectivement 9,6 et $9 \mathrm{~kg} / \mathrm{tête} / \mathrm{an}$, mais ces données moyennes sont quelque peu faussées par l'Afrique du Sud (14 $\mathrm{kg}$ ) et le Sénégal, ce dernier pays ayant une consommation atypique de corps gras (20 kg).

Ces réserves nous incitent à rappeler que ces moyennes nationales ou régionales masquent à l'évidence de profondes inégalités de la demande émanant des différents groupes sociaux ou des différentes régions d'un pays donné.

L'Afrique de l'Ouest côtière est caractérisée à la fois par le niveau élevé de ses disponibilités totales en corps gras alimentaires $(48,4 \%$ du total de l'ASS, alors que sa population n'en représente que 30 $\%, 50,7 \%$ des huiles végétales et $65,6 \%$ de l'huile de palme). Entre les huit pays composant cette zone, on observe également une grande hétérogénéité, les disponibilités par tête évoluant entre 5,3 (Bénin) et 16,6 kg/tête (Nigeria). C'est aussi la grande région de production et de consommation de I'huile de palme, avec l'Afrique centrale.

C'est le rôle central de l'huile de palme qu'il convient d'analyser plus en détail.

Place de l'huile de palme dans l'approvisionnement en corps gras de l'ASS

Comme le montre le tableau 3, I'huile de palme (à usage alimentaire) représentait, en 1995/1997, $36,3 \%$ des disponibilités totales en corps gras alimentaires de I'ASS et près de $39 \%$ des huiles végétales. Mais cette image, quelque peu statique, doit être mise en perspective car on est en présence d'une évolution aussi rapide que durable. 
En effet, la demande mondiale d'huile de palme, sous l'impulsion de la Malaisie, a vu son statut évoluer radicalement depuis 30 ans. Corps gras secondaire jusqu'à la fin des années 70, I'huile de palme est aujourd'hui la deuxième huile produite et la première huile exportée dans le monde. Elle est désormais consommée dans des pays qui l'ignoraient totalement dans les années 60. Et l'Afrique subsaharienne n'a pas échappé à ce phénomène puisque trente pays n'en consommaient pas au début des années 60 contre cinq à la fin des années 90 . La mise en parallèle des disponibilités par tête en huile de palme en 1961/1963 et en 1996/1998, pour les six grandes zones identifiées est, de ce point de vue, éloquente. Le tableau 4 (incluant également l'Afrique du Nord) résume ces disponibilités, tous usages confondus (en kg/tête/an).

Alors que les zones de production traditionnelles ne connaissent qu'une croissance faible de leurs disponibilités (Afrique centrale), voire un tassement (Afrique de l'Ouest), toutes les autres deviennent progressivement consommatrices d'huile de palme, importée d'Asie pour l'essentiel. Des pays, comme le Kenya ou l'Afrique du Sud, sont ainsi devenus en une décennie des importateurs d'huile de palme significatifs à l'échelle mondiale même si, pour ces pays, l'utilisation industrielle (savonnerie) l'emporte sur la consommation humaine. Des prix attractifs par rapport aux huiles de soja ou de tournesol, une offre diversifiée (huile brute ou raffinée), des produits de qualité, une agressivité commerciale permanente des exportateurs asiatiques sont quelques-unes des raisons pouvant expliquer l'ampleur et la rapidité de cette évolution. Mais l'explication principale réside sans doute dans l'attitude des grands pays producteurs africains (et leurs difficultés propres, aussi nombreuses que variées) qui, lorsqu'ils exportaient, visaient le marché européen et négligeaient, à quelques rares exceptions près, un marché africain qui s'ouvrait largement à l'huile de palme.

Les tableaux 5 et 6 synthétisent cette évolution depuis 1961/1965 sur la base de moyennes quinquennales. Calculés à la fois pour l'Afrique OUA et pour l'Afrique subsaharienne, à l'image des bilans des tableaux 1 et 2 , ces tableaux mettent en évidence :

- la faiblesse de la croissance de la production d'huile de palme (1,7 \% I'an contre 13,7\% en Malaisie)

- la croissance rapide des importations ${ }^{6}$, presque inexistantes en début de période (+ 13,3\% par an), et la chute des exportations $(-1,7 \%)$ qui, conjointement, font passer le solde des échanges de +307 000 tonnes à -525000 tonnes $^{7}$, tout en permettant au disponible d'augmenter de 3,6 \% l'an ;

- la dépendance de la demande vis-à-vis des importations, quasi nulle dans les années 60 , atteint 30 $\%$ en fin de période ;

- le disponible moyen par tête de l'Afrique subsaharienne augmente légèrement $(3,7$ contre 2,9 $\mathrm{kg} / \mathrm{an}$, soit $+0,7 \%$ l'an) mais, portant sur plus de 622 millions de consommateurs, cet accroissement représente un volume qui est loin d'être négligeable à l'échelle mondiale (1,6 million de tonnes).

Bien qu'étant, historiquement, le principal pourvoyeur de corps gras en Afrique subsaharienne, le palmier à huile a donc très mal résisté à l'irruption des grands producteurs asiatiques sur un marché mondial que ces derniers ont créé de toutes pièces et à qui ils ont insufflé un réel dynamisme. Non seulement, la Malaisie et I'Indonésie se sont emparés de leurs marchés de proximité particulièrement porteurs ${ }^{8}$, mais ils sont désormais présents dans le monde entier et... en Afrique. 
La prévision de l'évolution à long terme des multiples composantes d'une demande de corps gras si mal connue est un exercice hautement aléatoire qui, de surcroît, sort du cadre de cet article, si on entend la traiter de manière approfondie. Cependant, le manque de perspective et l'absence de vision stratégique de l'évolution de la demande de corps gras à long terme ont été soulignés avec suffisamment d'insistance pour que ne soient pas esquissées, à des fins essentiellement " pédagogiques ", des projections construites sur des hypothèses simples.

On proposera ainsi un scénario suivant lequel la production totale de corps gras poursuivra sa croissance observée de 1961/1965 à 1996/1998 ${ }^{9}$, soit 2,3 \% I'an, et ceci sans volontarisme particulier des pouvoirs publics et sans stratégie sous-régionale ou continentale clairement exprimée. Implicitement, cette hypothèse suppose que ce sont les producteurs, organisés ou non, qui assumeront la responsabilité de cet objectif de croissance. Par ailleurs, il est supposé que la croissance démographique de l'ASS ralentira pour s'établir à 2,5\% par an, en moyenne, durant les 20 prochaines années. Enfin, il est estimé que le disponible par tête, tous corps gras et tous usages confondus, continuera à croître de 0,9\% par an pour atteindre 10 kg/an en 2020.

Les résultats des projections, avec mise à jour des ratios caractéristiques déjà utilisés, figurent dans le tableau 7. Ils montrent que la réalisation simultanée des trois hypothèses retenues aurait pour conséquence d'accroître le déficit en corps gras de l'ASS (3,8 millions de tonnes, si l'on suppose que les exportations disparaissent, davantage si elles subsistent) et de porter son taux de dépendance vis-à-vis du reste du monde à près de $35 \%$. Si la production n'est pas en mesure de renouveler ses performances passées et si la population croît plus vite que prévu - hypothèses à ne pas exclure - le déficit se creuserait mécaniquement ${ }^{10}$ et il y a à craindre que le relèvement envisagé du disponible par tête, pourtant modeste, ne puisse se réaliser ${ }^{11}$.

Les résultats de ce scénario, pourtant proche du statu quo, devraient constituer une incitation forte à une " mobilisation générale " des divers acteurs impliqués, notamment dans les zones offrant un potentiel oléagineux reconnu. Mais à l'heure où, de manière générale, le démantèlement des filières agricoles semble l'emporter largement sur leur développement, il est difficile d'affirmer que cette voie sera privilégiée par les parties concernées.

Le même exercice, limité à la seule huile de palme en Afrique subsaharienne ${ }^{12}$, tel que décrit dans le tableau 8, fournit d'autres motifs d'inquiétude. D'une part, on peut s'interroger sur la capacité des pays producteurs d'huile de palme à maintenir un taux de croissance de 1,7 \% par an avec des plantations vieillissantes dont le renouvellement est loin d'être systématique ou d'atteindre l'ampleur souhaitable et, d'autre part, même si cette condition est remplie, le passage à $6 \mathrm{~kg}$ d'huile de palme par tête et par an aurait pour effet de générer un énorme déficit approchant les 4 millions de tonnes par an en 2020. Or, on peut penser que, dans les pays non producteurs d'huile de palme, la demande continuera à augmenter alors que, chez ceux qui en produisent, il sera sans cesse plus difficile de maintenir la demande intérieure à son niveau actuel.

On pourrait multiplier les scénarios à l'infini, les uns plus optimistes, les autres plus pessimistes ; tous montreraient que l'Afrique, seul continent dont la population sera encore majoritairement agricole au $x{ }^{i}{ }^{e}$ siècle, aura de plus en plus de difficultés à satisfaire ses besoins en corps gras. Sans prôner pour autant une stricte autosuffisance sur une base nationale, concept pour le moins douteux au nom duquel trop d'erreurs coûteuses ont été commises, il reste que l'Afrique subsaharienne a un potentiel oléagineux, inégalement réparti, souvent exagéré, mais réel. Même si la coopération 
internationale privilégie désormais des concepts de développement plus abstraits (pauvreté, genre, droits de l'homme, démocratie), il n'est pas inutile de rappeler que produire plus et, si possible, produire mieux peut constituer un slogan au moins aussi mobilisateur.

En 1995, une étude conjointe AFD/CIRAD, déjà évoquée par $O C L^{13}$, avait essayé de montrer que l'absence d'une vision claire de l'enjeu que représente le palmier, le manque d'ambition des stratégies des principaux producteurs africains et le vieillissement de leurs plantations risquaient d'entraîner la disparition du palmier à huile cultivé sur le continent. Dans la même étude, l'examen des perspectives des autres cultures oléagineuses, pérennes et annuelles, concluait sur la quasiimpossibilité de ces cultures à se substituer au palmier dans des conditions agronomiques et économiques satisfaisantes.

Cinq ans plus tard, le message ne semble avoir été entendu ni par les " décideurs ", ni par les bailleurs de fonds, ni par les sociétés de plantation ${ }^{14}$. En revanche, un engouement certain des petits et moyens planteurs pour le palmier à huile est observable dans des pays comme le Cameroun ${ }^{15}$ ou la Côte d'Ivoire. Reposant sur des motivations diverses (appropriation foncière, financement de la retraite, remplacement de cultures de rente en crise - cacao au Cameroun), ce mouvement est très mal connu (il n'utilise pas les circuits financiers habituels) et n'est canalisé ni par des organisations professionnelles embryonnaires, ni par l'administration. Aussi sympathique que positif pour la filière huile de palme (et donc pour la satisfaction de la demande future), ce mouvement pourrait également se traduire par de nouvelles désillusions (qualité incertaine des réalisations et surtout du matériel végétal utilisé) et par des relations conflictuelles avec les grandes sociétés publiques et privées du secteur formel.

De la capacité des administrations et des bailleurs de fonds, aujourd'hui quelque peu désemparés face à ce mouvement qui leur échappe, à définir un nouveau cadre institutionnel dans lequel pourront s'insérer ces nouveaux acteurs et à mettre au point des instruments financiers adaptés dépendront la relance du palmier à huile en Afrique subsaharienne et donc la satisfaction de la demande à long terme de corps gras. Si tel ne devait pas être le cas, ce sont les producteurs asiatiques, encouragés par l'inéluctable ouverture des frontières, qui capteront un marché que l'Afrique aurait pu ne jamais perdre. Même si la Malaisie "découvre » à son tour ${ }^{16}$ que ses plantations ont vieilli et que le rythme insuffisant des replantations, depuis quelques années, risque d'entraîner une stagnation, voire une baisse de la production d'huile de palme d'ici à 2006/2008, mettant ainsi fin à une période de croissance quasi ininterrompue de 30 ans.

Notes

${ }^{1}$ Qui peut, par exemple, privilégier le maintien d'un flux d'exportation au détriment de la demande intérieure (cas du Sénégal).

${ }^{2}$ Selon Oil World, la consommation par tête de ces quatre pays a évolué comme suit de 1976 à 1998 : 
Chine $\quad 2,7 \mathrm{~kg} \quad 12,8 \mathrm{~kg} \quad+379 \%$

Indonésie 4,8 $\mathrm{kg} \quad 14,8 \mathrm{~kg} \quad+210 \%$

Brésil $\quad 12,0 \mathrm{~kg} 24,1 \mathrm{~kg} \quad+101 \%$

Mexique $9,2 \mathrm{~kg} \quad 22,6 \mathrm{~kg} \quad+146 \%$

Afrique $\quad 6,2 \mathrm{~kg} \quad 7,9 \mathrm{~kg} \quad+27 \%$

subsaharienne

${ }^{3}$ Seuil au demeurant difficile à quantifier : les plus gros consommateurs de corps gras sont les ÉtatsUnis, avec plus de 49 kg/tête (usage non alimentaire inclus) devant l'Union européenne (45 kg).

${ }^{4}$ Ces six régions sont l'Afrique australe, I'Afrique centrale, I'Afrique de l'Est, l'Afrique de l'Ouest côtière, l'Afrique de l'Ouest sahélienne et l'océan Indien.

${ }^{5}$ L'Éthiopie et l'Ouganda, avec respectivement 2,5 et 2,4 kg/tête/an, tirent d'autant plus vers le bas la moyenne régionale qu'ils représentent $56,5 \%$ de la population. À l'inverse, Djibouti dispose de 15,7 $\mathrm{kg} /$ tête et se situe au-dessus de la moyenne de l'ASS. Pour l'océan Indien, l'éventail est encore plus ouvert puisque Maurice dispose de 22,5 kg, alors que Madagascar n'en a que 3,8 mais pèse de tout son poids démographique ( $85 \%$ ) sur la moyenne de la zone.

${ }^{6}$ Qui sont surtout le fait des pays non producteurs dont les importations représentent, en Afrique subsaharienne, environ $65 \%$ des importations totales. Mais l'ouverture croissante des frontières pourrait remettre en cause ce partage, notamment en raison de la forte demande des savonniers.

${ }^{7}$ Soit $61 \%$ du solde des échanges tous corps gras confondus (tableau 1 et 2).

${ }^{8}$ Chine, Inde et Pakistan sont les trois pays important plus d'un million de tonnes d'huile de palme par an.

${ }^{9}$ Cf. tableau 2.

${ }^{10}$ Cinq millions de tonnes, si la production n'augmente que de $2 \%$ par an et si la population croît de $2,75 \%$ I'an.

${ }^{11}$ Son maintien à $8,2 \mathrm{~kg} / \mathrm{tête} / \mathrm{an}$, toutes choses égales d'ailleurs, ramènerait le déficit à 1,8 million de tonnes.

${ }^{12}$ Avec les mêmes hypothèses auxquelles on ajoute une part croissante de l'huile de palme, qui passe de $50,4 \%$ du disponible à $60 \%$ en 2020 .

${ }^{13}$ L'huile de palme dans les pays du golfe de Guinée et les besoins en replantation à long terme, mai 1995, 210 pages, 2 volumes. Voir OCL (1996), 3, 2 : 98-104. 
${ }^{14}$ Du moins, par une majorité d'entre elles.

${ }^{15}$ Voir article sur la « Dynamique récente des plantations individuelles de palmier à huile au Cameroun » dans ce même numéro.

${ }^{16}$ Oil World Weekly (2000), 43, $11: 111-3$.

Illustrations

\begin{tabular}{|c|c|c|c|c|c|c|c|c|c|}
\hline Paramètres & $\begin{array}{l}\text { Moyenne } \\
1961 / 1965\end{array}$ & $1966 / 1970$ & $\begin{array}{l}\text { Moyenne } \\
\text { 1971/1975 }\end{array}$ & $\begin{array}{l}\text { Moyenne } \\
1976 / 1980\end{array}$ & $\begin{array}{l}\text { Moyenne } \\
1981 / 1985\end{array}$ & $\begin{array}{l}\text { Moyenne } \\
\text { 1986/1990 }\end{array}$ & $\begin{array}{l}\text { Moyenne } \\
1991 / 1995\end{array}$ & $\begin{array}{l}\text { Moyenne } \\
\text { 1996/1998 }\end{array}$ & $\begin{array}{c}\text { Taux } \\
\text { de croissance } \\
\text { annuel } \\
1961 / 1965 \text { - } \\
1996 / 1998\end{array}$ \\
\hline Importatiors (ton nes) & 360100 & 527200 & 757200 & 1319000 & 2111400 & 2453200 & 3014800 & 3362500 & $6,8 \%$ \\
\hline Production (tonnes) & 2434191 & 2095056 & 3069565 & 3561558 & 3458375 & 4124547 & 4728334 & 5428460 & $2,4 \%$ \\
\hline Exportations (tonnes) & 662000 & 692400 & 665200 & 536200 & 412000 & 478000 & 606800 & 576000 & $-0,4 \%$ \\
\hline Disponible (tonnes) & 2132291 & 2529856 & 3161565 & 4299358 & 5157775 & 6099747 & 7136334 & 8214960 & $4,0 \%$ \\
\hline Population (milliers) ${ }^{\circ}$ & 289309 & 328665 & 380114 & 437209 & 517100 & 595000 & 682000 & 759300 & $2,9 \%$ \\
\hline \multicolumn{10}{|l|}{ Ratios } \\
\hline Import/dis pon ible (\$) & 16,9 & 20,8 & 24,0 & 30,7 & 40,9 & 40,2 & 42,2 & 40,9 & \\
\hline Exports/production (\%) & 27,2 & 25,7 & 21,7 & 15,2 & 11,9 & 11,6 & 12,8 & 10,6 & \\
\hline Taux d'autosuffisa nce (\%) & 114,2 & 106,5 & 97,1 & 81,8 & 67,1 & 67,6 & 66,3 & 66,1 & \\
\hline Disponible/tête (kg/an) & 7,4 & 7,7 & 8,3 & 9,8 & 10,0 & 10,3 & 10,5 & 10,8 & $1,1 \%$ \\
\hline Solde échanges (tonnes) & 301900 & 165200 & -92000 & -782800 & -1699400 & -1975200 & -2408000 & -2786500 & \\
\hline
\end{tabular}

Tableau 1. Afrique OUA, bilan corps gras végétaux et animaux 


\begin{tabular}{|c|c|c|c|c|c|c|c|c|c|}
\hline Paramètres & $\begin{array}{l}\text { Moyenne } \\
1961 / 1965\end{array}$ & $1966 / 1970$ & $\begin{array}{l}\text { Moyenne } \\
1971 / 1975\end{array}$ & $\begin{array}{l}\text { Moyenne } \\
\text { 1976/1980 }\end{array}$ & $\begin{array}{l}\text { Moyenne } \\
1981 / 1985\end{array}$ & $\begin{array}{l}\text { Moyenne } \\
1986 / 1990\end{array}$ & $\begin{array}{l}\text { Moyenne } \\
1991 / 1995\end{array}$ & $\begin{array}{l}\text { Moyenne } \\
\text { 1996/1998 }\end{array}$ & $\begin{array}{c}\text { Taux } \\
\text { de croissance } \\
\text { annuel } \\
1961 / 1965- \\
1996 / 1998\end{array}$ \\
\hline Importatiors (tonnes) & 95402 & 129919 & 159072 & 415337 & 695669 & 725129 & 987981 & 1259282 & $7,9 \%$ \\
\hline Production (tonnes) & 1950372 & 2152801 & 2305327 & 2588904 & 2577442 & 3189179 & 3712179 & 4238926 & $2,3 \%$ \\
\hline Exportations (tonnes) & 624943 & 617982 & 548167 & 440663 & 314359 & 389464 & 417758 & 399897 & $-1,3 \%$ \\
\hline Disponible (tonnes) & 1420831 & 1664737 & 1916232 & 2563578 & 2958752 & 3624844 & 4283079 & 5098311 & $3,8 \%$ \\
\hline Population (milliers) & 233970 & 270420 & 312820 & 362250 & 418770 & 483630 & 556430 & 622670 & $2,9 \%$ \\
\hline \multicolumn{10}{|l|}{ Ratios } \\
\hline Imports/dis ponible (\%) & 6,7 & 7,8 & 8,3 & 16,2 & 23,5 & 20,6 & 23,1 & 24,7 & \\
\hline Exports/production (\%) & 32,0 & 28,7 & 23,8 & 17,0 & 12,2 & 12,2 & 11,3 & 9,4 & \\
\hline Taux d'autosuffisance (\$) & 137,3 & 129,3 & 120,3 & 101,0 & 87,1 & 90,5 & 86,7 & 83,1 & \\
\hline Disponible/tête (kg/an) & 6,1 & 6,2 & 6,1 & 7,1 & 7,1 & 7,3 & 7,7 & 8,2 & $0,9 \%$ \\
\hline Solde échanges (tonnes) & 529541 & 488064 & 389095 & 25326 & -381310 & -335665 & -570222 & -859385 & \\
\hline
\end{tabular}

Tableau 2. Afrique subsaharienne, bilan corps gras végétaux et animaux

\begin{tabular}{|c|c|c|c|c|c|c|c|c|}
\hline \multirow[t]{2}{*}{ Zone } & \multicolumn{2}{|c|}{ Population 1995/1997 } & \multicolumn{2}{|c|}{$\begin{array}{c}\text { Dispon ibilités } \\
\text { en corps gras alim. }\end{array}$} & \multicolumn{2}{|c|}{$\begin{array}{l}\text { Dispon ibilités } \\
\text { en huiles vég étales alim. }\end{array}$} & \multicolumn{2}{|c|}{$\begin{array}{l}\text { Dispon ibilités } \\
\text { en huile de palme alim. }\end{array}$} \\
\hline & Millions & $\%$ & 1000 tonn es & $\%$ & 1000 tonn es & $\%$ & 1000 tonnes & $\%$ \\
\hline Afrique australe & 104,31 & 18,6 & 997,9 & 20,1 & 873,1 & 18,9 & 76,1 & 4,2 \\
\hline Afrique centrale & 83,33 & 14,9 & 471,2 & 9,5 & 454,2 & 9,8 & 255,6 & 14,2 \\
\hline Afrique de l'Est & 146,13 & 26,1 & 624,6 & 12,6 & 521,3 & 11,3 & 234,4 & 13,0 \\
\hline Afrique Ouest côtère & 166,87 & 29,8 & 2404,3 & 48,4 & 2349,9 & 50,7 & 1182,6 & 65,6 \\
\hline Afrique Ouest Sahel & 42,50 & 7,6 & 380,0 & 7,7 & 357,5 & 7,7 & 44,2 & 2,4 \\
\hline Océan Indien & 16,66 & 3,0 & 87,2 & 1,8 & 75,0 & 1,6 & 9,9 & 0,5 \\
\hline Total & 559,80 & 100 & 4966,1 & 100 & 4631,1 & 100 & 1802,8 & 100 \\
\hline \multirow[t]{2}{*}{ Zone } & & & \multicolumn{2}{|c|}{ Dispon ibilités par tête } & \multicolumn{2}{|c|}{ Dont huiles végétales } & \multicolumn{2}{|c|}{ Dont huile de palme } \\
\hline & & & \multicolumn{2}{|c|}{$\mathrm{Kg} / \mathrm{an}$} & $\mathrm{Kg} /$ an & $\%^{\mathrm{a}}$ & $\mathrm{Kg} /$ an & $\% a$ \\
\hline Afrique australe & & & \multicolumn{2}{|c|}{9,57} & 8,37 & 87,5 & 0,73 & 7,6 \\
\hline Afrique centrale & & & \multicolumn{2}{|c|}{5,65} & 5,45 & 96,4 & 3,07 & 54,3 \\
\hline Afrique de l'Est & & & \multicolumn{2}{|c|}{4,27} & 3,57 & 83,5 & 1,60 & 37,5 \\
\hline Afrique Ouest côtière & & & \multicolumn{2}{|c|}{14,41} & 14,08 & 97,7 & 7,09 & 49,2 \\
\hline Afrique Ouest Sahel & & & \multicolumn{2}{|c|}{8,97} & 8,41 & 93,8 & 1,04 & 11,6 \\
\hline Océan Indien & & & \multicolumn{2}{|c|}{5,24} & 4,50 & 85,9 & 0,60 & 11,4 \\
\hline Moyenne & & & \multicolumn{2}{|c|}{8,87} & 8,27 & 93,3 & 3,22 & 36,3 \\
\hline
\end{tabular}

Tableau 3. Afrique subsaharienne : disponibilités totales et par tête en corps gras alimentaires, en huiles végétales et en huile de palme, par grande zone (moyenne 1995/1997)

${ }^{\text {a }}$ En \% des disponibilités totales par tête. 


\begin{tabular}{|lccc}
\hline Zon e & $1961 / 1963$ & $1996 / 1998$ & Variation (\%) \\
\hline Afrique australe & 0,23 & 1,89 & +722 \\
Afrique centrale & 3,18 & 4,22 & +33 \\
Afrique de l'Est & 0,07 & 1,75 & +2400 \\
Afr. de Youest côtière & 8,37 & 7,57 & -10 \\
Afr. de Youest sahélienne & 0,13 & 0,99 & +662 \\
Océan Indien & 0,00 & 0,63 & +6200 \\
Afrique subsaharienne & 2,98 & 3,70 & +24 \\
Afrique du Nord $(\mathrm{pm})$ & 0,01 & 2,47 & +24600 \\
\hline
\end{tabular}

Tableau 4. Afrique OUA : évolution des disponibilités par tête en huile de palme de 1961/1963 à 1996/1998, par grande zone

\begin{tabular}{|c|c|c|c|c|c|c|c|c|c|}
\hline Paramètres & $\begin{array}{l}\text { Moyenne } \\
1961 / 1965\end{array}$ & $1966 / 1970$ & $\begin{array}{l}\text { Moyenne } \\
197 / 1975\end{array}$ & $\begin{array}{l}\text { Moyenne } \\
\text { 1976/1980 }\end{array}$ & $\begin{array}{l}\text { Moyenne } \\
1981 / 1985\end{array}$ & $\begin{array}{l}\text { Moyenne } \\
\text { 1986/1990 }\end{array}$ & $\begin{array}{l}\text { Moyenne } \\
1991 / 1995\end{array}$ & $\begin{array}{l}\text { Moyenne } \\
\text { 1996/1998 }\end{array}$ & $\begin{array}{c}\text { Taux } \\
\text { de croissance } \\
\text { annuel } \\
1961 / 1965- \\
1996 / 1998\end{array}$ \\
\hline Importatiors (tonnes) & 10791 & 17536 & 34276 & 99951 & 290224 & 517000 & 993843 & 1054973 & $14,4 \%$ \\
\hline Production (tonnes) & 989156 & 923265 & 1107766 & 1320301 & 1395969 & 1615906 & 1750427 & 1776164 & $1,7 \%$ \\
\hline Exportations (tonnes) & 316208 & 198615 & 182328 & 111531 & 81023 & 147065 & 210879 & 174504 & $-1,7 \%$ \\
\hline Disponible (tonnes) & 683739 & 742186 & 959714 & 1308721 & 1605170 & 1985841 & 2533391 & 2656633 & $4,1 \%$ \\
\hline Population (milliers) ${ }^{a}$ & 289309 & 328665 & 380114 & 437209 & 517100 & 595000 & 682000 & 759300 & $2,9 \%$ \\
\hline \multicolumn{10}{|l|}{ Ratios } \\
\hline Import/dis ponible (\%) & 1,6 & 2,4 & 3,6 & 7,6 & 18,1 & 26,0 & 39,2 & 39,7 & \\
\hline Exports/ production (\%) & 32,0 & 21,5 & 16,5 & 8,4 & 5,8 & 9,1 & 12,0 & 9,8 & \\
\hline Taux d' autosuffisance (\$6) & 144,7 & 124,4 & 115,4 & 100,9 & 87,0 & 81,4 & $\theta 9,1$ & 66,9 & \\
\hline Disponible/tête (kg/an) & 2,4 & 2,3 & 2,5 & 3,0 & 3,1 & 3,3 & 3,7 & 3,5 & $1,2 \%$ \\
\hline Solde échanges (tonnes) & 305417 & 181079 & 148052 & 11580 & -209201 & -369935 & -782964 & -880469 & \\
\hline
\end{tabular}

Tableau 5. Afrique OUA : bilan huile de palme et ratios caractéristiques 


\begin{tabular}{|c|c|c|c|c|c|c|c|c|c|}
\hline Paramètres & $\begin{array}{l}\text { Moyenne } \\
1961 / 1965\end{array}$ & $\begin{array}{l}\text { Moyenne } \\
1966 / 1970\end{array}$ & $\begin{array}{l}\text { Moyenne } \\
1971 / 1975\end{array}$ & $\begin{array}{l}\text { Moyenne } \\
\text { 1976/1980 }\end{array}$ & $\begin{array}{l}\text { Moyenne } \\
1981 / 1985\end{array}$ & $\begin{array}{l}\text { Moyenne } \\
\text { 1986/1990 }\end{array}$ & $\begin{array}{l}\text { Moyenne } \\
1991 / 1995\end{array}$ & Moyenne & $\begin{array}{c}\text { Taux } \\
\text { de croissance } \\
\text { annuel } \\
1961 / 1965- \\
1996 / 1998\end{array}$ \\
\hline Importatiors (tonnes) & 9940 & 16667 & 33326 & 94104 & 260549 & 359713 & 590453 & 699119 & $13,3 \%$ \\
\hline Production (tonnes) & 989156 & 923265 & 1107766 & 1320301 & 1395969 & 1615906 & 1750427 & 1776164 & $1,7 \%$ \\
\hline Exportations (tonnes) & 316861 & 198597 & 182316 & 110757 & 81023 & 146998 & 210742 & 174338 & $-1,7 \%$ \\
\hline Dis ponible (tonnes) & 682235 & 741335 & 958776 & 1303648 & 1575495 & 1828621 & 2130138 & 2300945 & $3,6 \%$ \\
\hline Population (milliers) & 233970 & 270420 & 312820 & 362250 & 418770 & 483630 & 556430 & 622670 & $2,9 \%$ \\
\hline \multicolumn{10}{|l|}{ Ratios } \\
\hline Imports/dis ponible (\%) & 1,5 & 2,2 & 3,5 & 7,2 & 16,5 & 19,7 & 27,7 & 30,4 & \\
\hline Exports/production (\%) & 32,0 & 21,5 & 16,5 & 8,4 & 5,8 & 9,1 & 12,0 & 9,8 & \\
\hline Taux d'autosuffisance (\%) & 145,0 & 124,5 & 115,5 & 101,3 & 88,6 & 88,4 & 82,2 & 77,2 & \\
\hline Disponible/tête (kg/an) & 2,9 & 2,7 & 3,1 & 3,6 & 3,8 & 3,8 & 3,8 & 3,7 & $0,7 \%$ \\
\hline Solde échanges (tonnes) & 306291 & 181930 & 148990 & 16653 & -179526 & -212715 & -379711 & -524781 & \\
\hline
\end{tabular}

Tableau 6. Afrique subsaharienne, bilan huile de palme et ratios caractéristiques

\begin{tabular}{|c|c|c|c|c|c|}
\hline Paramètres & $\begin{array}{l}\text { Moyenne } \\
1961-1965\end{array}$ & $\begin{array}{l}\text { Moyenne } \\
1996-1998\end{array}$ & $\begin{array}{c}\text { Taux de croiss ance } \\
\text { an nuel } \\
1961 / 1965-1996 / 1998\end{array}$ & $\begin{array}{c}\text { Taux de croiss ance } \\
\text { an nuel } \\
1996 / 1998-2020\end{array}$ & $\begin{array}{c}\text { Horizon } 2020 \\
\text { tous corps } \\
\text { gras }\end{array}$ \\
\hline Importations (tonnes) & 95402 & 1259282 & $7,9 \%$ & $4,9 \%$ & 3820987 \\
\hline Production (tonnes) & 1950372 & 4238926 & $2,3 \%$ & $2,3 \%$ & 7166714 \\
\hline Exportations (tonnes) & 624943 & 399897 & $-1,3 \%$ & & 0 \\
\hline Disponible (tonnes) & 1420831 & 5098311 & $3,8 \%$ & $3,4 \%$ & 10987701 \\
\hline Population (milliers) ${ }^{a}$ & 233970 & 622670 & $2,9 \%$ & $2,50 \%$ & 1098770 \\
\hline \multicolumn{6}{|l|}{ Ratios } \\
\hline Imports/disponible (\%) & $6,7 \%$ & $24,7 \%$ & & & $34,8 \%$ \\
\hline Exports/production (\%) & $32,0 \%$ & $9,4 \%$ & & & $0,0 \%$ \\
\hline Taux d'auto suffisa nce (\%) & $137,3 \%$ & $83,1 \%$ & & & $65,2 \%$ \\
\hline Disponible/tête (kg/an) & 6,1 & 8,2 & $0,9 \%$ & $0,9 \%$ & 10,0 \\
\hline Solde échanges (tonnes) & 529541 & -859385 & & & -3820987 \\
\hline
\end{tabular}

Tableau 7. Projection de la demande globale de corps gras de l'Afrique subsaharienne

à l'horizon 2020 


\begin{tabular}{|c|c|c|c|c|c|}
\hline Paramètres & $\begin{array}{l}\text { Moyenne } \\
1961-1965\end{array}$ & $\begin{array}{l}\text { Moyenne } \\
1996-1998\end{array}$ & $\begin{array}{c}\text { Taux de croiss ance } \\
\text { an nuel } \\
1961 / 1965-1966 / 1998\end{array}$ & $\begin{array}{c}\text { Taux de croiss ance } \\
\text { an nuel } \\
1996 / 1998-2020\end{array}$ & $\begin{array}{c}\text { Horizon } 2020 \\
\text { tous corps } \\
\text { gras }\end{array}$ \\
\hline Importations (tonnes) & 9940 & 699119 & $13,3 \%$ & $7,8 \%$ & 3963531 \\
\hline Production (tonnes) & 989156 & 1776164 & $1,7 \%$ & $1,7 \%$ & 2639090 \\
\hline Exportations (tonnes) & 316861 & 174338 & $-1,7 \%$ & & 0 \\
\hline Disponible (tonnes) & 682235 & 2300945 & $3,6 \%$ & $4,7 \%$ & 6592621 \\
\hline Population (milliers) ${ }^{\mathrm{a}}$ & 233970 & 622670 & $2,9 \%$ & $2,50 \%$ & 1098770 \\
\hline \multicolumn{6}{|l|}{ Ratios } \\
\hline Imports/disponible (\%) & $1,5 \%$ & $30,4 \%$ & & & 60,0 \\
\hline Exports/production (\%) & $32,0 \%$ & $9,8 \%$ & & & 0,0 \\
\hline Taux d'auto suffisa nce (\%) & $145,0 \%$ & $77,2 \%$ & & & 40,0 \\
\hline Disponible/tête (kg/an) & 2,9 & 3,7 & $0,7 \%$ & $2,1 \%$ & 6,0 \\
\hline Solde échanges (tonnes) & 306921 & -524781 & & & -3953531 \\
\hline
\end{tabular}

Tableau 8. Projection de la demande globale d'huile de palme de l'Afrique subsaharienne à I'horizon 2020

NB : Les taux de croissance en gras constituent les hypothèses de base servant aux projections, les autres étant calculés.

Sources : FAOSTAT CDROM 1998 \& calculs.

${ }^{a}$ Année médiane. 